See discussions, stats, and author profiles for this publication at: https://www.researchgate.net/publication/313805746

\title{
Demo: HELICoiD tool demonstrator for real-time brain cancer detection
}

Conference Paper · October 2016

DOI: 10.1109/DASIP.2016.7853831

CITATIONS

11

8 authors, including:

Ruben Salvador

CentraleSupélec

48 PUBLICATIONS 373 CITATIONS

SEE PROFILE

Raquel Lazcano

31 PUBLICATIONS 170 CITATIONS

SEE PROFILE

Some of the authors of this publication are also working on these related projects:

Project HELICoiD: HypErspectraL Imaging Cancer Detection View project

Project [Helicoid] Hyperspectral imaging for brain cancer detection View project
READS

75

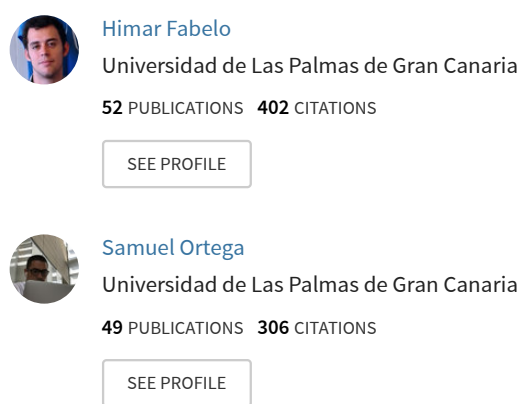




\title{
HELICoiD Tool Demonstrator for Real-Time Brain Cancer Detection
}

\author{
R. Salvador ${ }^{1}$, H. Fabelo ${ }^{2}$, R. Lazcano ${ }^{1}$, S. Ortega ${ }^{2}$, D. Madroñal ${ }^{1}$, G. M. Callicó ${ }^{2}$, E. Juárez ${ }^{1}$, C. Sanz ${ }^{1}$ \\ ${ }^{1}$ Centre of Software Technologies and Multimedia Systems (CITSEM), Technical University of Madrid (UPM), Spain \\ ${ }^{2}$ Research Institute for Applied Microelectronics (IUMA), University of Las Palmas de Gran Canaria (ULPGC), Spain \\ \{ruben.salvador, raquel.lazcano, daniel.madronal, eduardo.juarez, cesar.sanz\}@upm.es \\ \{hfabelo, sortega, gustavo\}@iuma.ulpgc.es
}

\begin{abstract}
In this paper, a demonstrator of three different elements of the EU FET HELICoiD project is introduced. The goal of this demonstration is to show how the combination of hyperspectral imaging and machine learning can be a potential solution to precise real-time detection of tumor tissues during surgical operations. The HELICoiD setup consists of two hyperspectral cameras, a scanning unit, an illumination system, a data processing system and an EMB01 accelerator platform, which hosts an MPPA-256 manycore chip. All the components are mounted fulfilling restrictions from surgical environments, as shown in the accompanying video recorded at the operating room. An in-vivo human brain hyperspectral image data base, obtained at the University Hospital Doctor Negrin in Las Palmas de Gran Canaria, has been employed as input to different supervised classification algorithms (SVM, RF, NN) and to a spatial-spectral filtering stage (SVM-KNN). The resulting classification maps are shown in this demo. In addition, the implementation of the SVM-KNN classification algorithm on the MPPA EMB01 platform is demonstrated in the live demo.
\end{abstract}

Keywords - Hyperspectral Imaging; Machine Learning; Supervised Classification; Massively Parallel Processing; Real Time Processing; KNN Filtering

\section{INTRODUCTION}

Hyperspectral Imaging (HI) collects a huge amount of spectral and spatial information from a scene so hundreds of bands covering a narrow and almost continuous portion of the spectrum conform each pixel. The information provided is usually structured as a data-cube where each plane consists of an image captured at a certain wavelength. Analyzing the datacube using machine learning techniques helps determining the type of material present at each pixel.

In 2012, the estimated number of cancer cases around the world, based on data from the World Cancer Research Fund (WCRF International), was of 14.1 million. By 2035, this number is expected to increase to 24 million. According to WCRF statistics, the most common cancer in the world is lung $(13.0 \%)$, followed by breast $(11.9 \%)$, colorectal $(9.7 \%)$ prostate $(7.9 \%)$ and stomach cancer $(6.8 \%)$. Hence, cancer is a world growing clinical burden.
Currently, the main tool medical practitioners have for differentiating normal from malignant tissues remains the educated human eye. Although other techniques have been developed, none of them has succeeded in reliable tissue differentiation. Neuronavigation is plagued by brain shift, ultrasound is highly operator dependent and Magnetic Resonance Imaging (MRI) fails to provide real time images, obtaining just a few occasional snapshots during surgery. Under these circumstances, the combination of $\mathrm{HI}$ and Machine Learning (ML) arises as a potential solution that allows precise real-time detection of malignant tissues during surgical interventions. In addition, it could also be used as an assisting tool for clinical diagnosis. Furthermore, it is worth noting that $\mathrm{HI}$ is a minimally invasive sensing technique.

In this context, the main goal of the European FET project HELICoiD (HypErspectraL Imaging Cancer Detection) [1] is to develop an experimental intraoperative setup based on hyperspectral cameras connected to a massively parallel processing platform running a set of algorithms capable of precise real-time discrimination between healthy and pathological tissues during neurosurgical operations. This information is provided to the surgeon through a dedicated display device, overlapping normal viewing images of the surgical scene with simulated color images that indicate the presence or absence of cancer in the exposed tissue.

By means of the HELICoiD setup, neurosurgeons will have a new tool to make precise decisions during brain tumor resection. Complete resection is of paramount importance to improve surgery results to ameliorate the quality of life of brain tumor patients. However, to achieve this goal, a correct diagnose of the malignant region is required. Currently, diagnoses of internal tumors are based on excisional biopsy followed by histology analysis. This technique suffers from two issues. First, its aggressiveness has potential complications due to the surgical resection of both tumor and healthy tissues. Second, the diagnose results are not available in real time since tissues have to be processed in a laboratory. As a consequence, the prospect precision of real-time detection of malignant tissues during surgical procedures using HELICoiD tools contributes to attain the aim of complete resection. 


\section{HELICOID SETUP}

The HELICoiD setup consists of two hyperspectral cameras. The first of them works in the visible and near infrared spectral range $(400-1000 \mathrm{~nm})$ and the second in the near infrared range $(900-1700 \mathrm{~nm})$. These cameras are attached to a push-broom scanning unit. The illumination system provides, through an optical fiber connected to a quartz tungsten-halogen lamp, a cold light from 400 to $2200 \mathrm{~nm}$. This structure isolates the heat generated by the lamp from the brain surface. A data processing unit (DPU) manages the setup providing the hyperspectral images to an embedded system, the EMB01 platform, which contains a 256-core Massively Parallel Processor Array (MPPA) as an accelerator to speed up classification algorithms. To ease moving this setup in the operating theatre, the different components are mounted in a specific structure for surgical environments.

\section{A. The MPPA EMB01 Kalray Platform}

The processing platform deployed in the operating room is the MPPA EMB01 board, which contains a standard host x86 ComExpress module working as an embedded computer, and a carrier board containing the MPPA manycore chip. The MPPA EMB01 board is connected through an Ethernet socket with the DPU. The host side has a CT-CA564 ComExpress module with an AMD G-T40E Dual Core Processor running CentOS 7 GNU/Linux OS, 4GB DDR3 RAM memory, 1 PCIe Gen2x2 for communication with the MPPA and 16 GB SSD as system disk. The carrier board consists of an MPPA-256 manycore processor, 4GB of DDR3 memory and the host PCIe to MPPA Gen2x2 port. The MPPA-256 consists of 256 cores structured in 16 compute clusters with $2 \mathrm{MB}$ local memory each. In addition, 2 quad-core I/O subsystems manage the communication between the cluster cores and the external memory. A Network-on-Chip (NoC) controls the communication among the clusters and the I/O subsystems.

\section{B. Hyperspectral Image Dataset}

An in-vivo human brain hyperspectral image database has been obtained with the HELICoiD setup from 22 different patients at the University Hospital Doctor Negrin. Primary brain tumors and secondary tumors (metastasis) have been found. For primary tumors, the diagnoses have been glioblastoma, ganglioglioma and meningioma. In secondary tumors, lung, renal and breast metastasis have been identified. To label these samples, which will be used to build the training set of the classification algorithms, neurosurgeons remove the tissue inside two makers - rubber rings - that have been placed over the surface of the exposed brain. One of the markers is placed in an area where the tumor is clearly found; the other in a position where neurosurgeons are confident that brain tissue is healthy. The ground-truth is given by the pathologist, who specifies the grade and type of tumor of each sample. The corresponding pixels, now correctly labeled, are used as the training inputs to the supervised classification algorithms.

\section{DEMO DESCRIPTION}

The demonstrator of the HELICoiD project consists of three different elements.

\section{A. HELICoiD setup}

The HELICoiD setup working in the operating room at the University Hospital Doctor Negrin in Las Palmas de Gran Canaria, Spain, is shown in a video that explains the procedure followed to capture different hyperspectral data-cubes of a patient during the surgical resection of a brain tumor.

\section{B. Classification Algorithms}

Second, functional results of supervised classification algorithms using the hyperspectral images captured is shown. The algorithms used are: Support Vector Machines (SVM); Random Forest (RF); Neural Networks (NN); and K-Nearest Neighbours (KKN) [2] to increase spatial coherence. After the acquisition step, a preprocessing chain is applied to the labelled dataset. Before the actual classification stage, the labeled dataset is partitioned into two new different datasets using three-way cross-validation. One, the training dataset, is employed to obtain the classifier model. The other, the test dataset, is used to assess the quality of the obtained classifier. The metrics selected to evaluate the classifier results are sensitivity, specificity and overall accuracy. At last, the resulting classification maps are shown.

\section{MPPA EMB01 Implementation}

Lastly, the implementation of the SVM-KNN classification algorithm in the MPPA EMB01 platform is demonstrated using real hyperspectral images captured at the operating room. The probability map provided by the SVM classification algorithm, the projection of the hyperspectral data-cubes on the first principal component and the averaged probability map obtained after KNN-based filtering will be jointly shown with the resulting classification maps [2].

\section{CONCLUSION}

The demonstrator shows the possibility to provide neurosurgeons with a tool to make precise real-time decisions during brain tumor resection. This tool will contribute to improve surgery results and, as a consequence, the quality of life of patients after surgery. Classification maps from the different machine learning algorithms evaluated, as well as implementation results on a manycore platform, when in-vivo human brain hyperspectral images are employed as inputs, are shown as evidences of the tool potential.

\section{ACKNOWLEDGMENT}

This work has been party supported by the European Commission through the FP7 FET Open programme ICT2011.9.2, European Project HELICoiD HypErspectral Imaging Cancer Detection under Grant Agreement 618080.

\section{REFERENCES}

[1] HELICoiD (Hyperspectral Imaging Cancer Detection) project website (last access on 05/27/2016): http://www.helicoid.eu

[2] Huang, K., Li, S., Kang, X., \& Fang, L. (2016). Spectral-Spatial Hyperspectral Image Classification Based on KNN. Sensing and Imaging, 17(1), 1-13. 